\title{
Peer-Led, Empowerment-Based Approach to Self-Man- agement Efforts in Diabetes (PLEASED): A Randomized Controlled Trial in an African American Community
}

Tricia S. Tang, $P b D^{1}$

Martha M. Funnell, MS, RN, CDE

Brandy Sinco, MS ${ }^{3}$

Michael S. Spencer, PbD, MSW

Michele Heisler, MD, MPA ${ }^{4,5}$

'University of British Columbia Department of Medicine, Vancouver, British Columbia

${ }^{2}$ University of Michigan Department of Learning Health Sciences. Ann Arbor Michigan

${ }^{3}$ University of Michigan School of Social Work, Ann Arbor, Michigan

${ }^{4}$ University of Michigan Department of Internal Medicine, Ann Arbor, Michigan

${ }^{5}$ Ann Arbor VA Center for Clinical Management Research (CCMR), Ann Arbor, Michigan

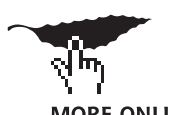

MORE ONLINE
www.annfammed.or
Conflicts of interest: The authors report none.

\section{CORRESPONDING AUTHOR}

Tricia S. Tang, PhD

University of British Columbia

2775 Laurel Street, 10th Floor

Vancouver, BC V5Z 1M9

tricia.tang@vch.ca

\begin{abstract}
PURPOSE We compared a 3-month diabetes self-management education (DSME) program followed by a 12-month peer support intervention with a 3-month DSME program alone in terms of initial and sustained improvements in glycated hemoglobin $\left(\mathrm{HbA}_{\mathrm{c}}\right)$. Secondary outcomes were risk factors for cardiovascular disease (CVD), diabetes distress, and social support.
\end{abstract}

METHODS We randomized 106 community-dwelling African American adults with type 2 diabetes to a 3-month DSME program followed by 12 months of weekly group sessions and supplementary telephone support delivered by peer leaders or to a 3-month DSME program with no follow-up peer support. Assessments were conducted at baseline, 3, 9, and 15 months.

RESULTS No changes in $\mathrm{HbA}_{1 \mathrm{c}}$ were observed at 3 months or at 15 months for either group. The peer support group either sustained improvement in key CVD risk factors or stayed the same while the control group worsened at 15 months. At 15 months, the peer-support group had significantly lower low-density lipoprotein cholesterol levels $(-15 \mathrm{mg} / \mathrm{dL}, P=.03)$, systolic blood pressure $(-10 \mathrm{~mm}$ $\mathrm{Hg}, P=.01)$, diastolic blood pressure $(-8.3 \mathrm{~mm} \mathrm{Hg}, P=.001)$, and body mass index $\left(-0.8 \mathrm{~kg} / \mathrm{m}^{2}, P=.032\right)$ than the DSME-alone group.

CONCLUSIONS In this population of African American adults, an initial DSME program, whether or not followed by 12 months of peer support, had no effect on glycemic control. Participants in the peer-support arm of the trial did, however, experience significant improvements in some CVD risk factors or stay approximately the same while the control group declined.

Ann Fam Med 2015;13(Suppl_1):S27-S35. doi: 10.1370/afm.1819.

\section{INTRODUCTION}

W ith 13\% of African Americans known to have diabetes, African American adults bear the second greatest burden of diabetes among ethnic groups in the United States. ${ }^{1}$ Compared with their white counterparts, African American adults have worse blood glucose, blood pressure, and lipid control. ${ }^{2-5}$ They often face significant challenges in following recommendations for screening, diet, and physical activity. ${ }^{6}$ Effective diabetes self-management interventions are critically needed for this population.

Over the past decade, peer support has shown promise as a low-cost and culturally sensitive approach to improving self-management efforts in diabetes. Although meta-analyses and systematic reviews of peer support interventions have reported favorable clinical and mental health outcomes ${ }_{1}^{7-8}$ this model has not been widely tested among African Americans. In fact, only 3 of 14 randomized controlled trials analyzed in a 2012 systematic review of peer support interventions in diabetes targeted the African American community. ${ }^{7,9-11}$ While the few peer support trials in the African American community have generally yielded positive findings, ${ }^{10-13}$ the majority of 
these interventions have been short-term rather than longer-term diabetes self-management support (DSMS) programs. Accordingly, the objective of this study, the Peer-Led, Empowerment-Based Approach to SelfManagement Efforts in Diabetes (PLEASED) Study, was to investigate whether a peer support model could sustain improvements achieved in a short-term diabetes self-management education (DSME) program for African American adults with type 2 diabetes.

\section{METHODS}

\section{Setting and Identification of Patients}

This study, which was approved by the University of Michigan Institutional Review Board, was conducted in partnership with the Ann Arbor Community Center, Ann Arbor, and Parkside Community Center, Ypsilanti. From February 2009 to November 2011, we recruited participants by posting flyers in local community centers, businesses, and clinics; presenting at local African American churches; encouraging word-of-mouth dissemination; and acting on referrals from health care providers. To be eligible, individuals had to have type 2 diabetes, be at least 21 years of age, have a regular health care provider, and self-identify as African American.

\section{Recruitment and Randomization}

Potential participants called a toll-free number to undergo eligibility screening. Those deemed eligible attended an orientation session to learn more about the study and, if interested in enrolling, followed informed consent procedures and completed a baseline assessment that included a blood sample, blood pressure (BP), height, weight, body mass index (BMI), waist circumference measurements, and a self-report survey. Participants were then randomized to 1 of 2 arms: a 3-month DSME program followed by 12 months of peer-led DSMS, or a 3-month DSME program alone.

Random sequence generation and group assignment were determined centrally just prior to the initial session. Participants and staff were blinded to randomization results until completion of baseline assessment. Data assessors remained blinded to group assignment throughout the study. Participants received a stipend of $\$ 40$ upon completion of each of the 4 assessmentsbaseline, 3-month, 9-month, and 15-month.

\section{Description of Intervention}

\section{3-Month Diabetes Self-Management Education} Program

Both intervention and control groups received a 3-month DSME program delivered by a certified diabetes educator and 2 peer leaders (PLs). A detailed description of PL recruitment, selection and training and the diabetes educator's background are reported elsewhere. ${ }^{14}$ The 3 -month program consisted of 12 weekly 90-minute group sessions. The diabetes educator was responsible for delivering diabetes education while the 2 PLs directed behavior change activities. In the first session, each participant received a personalized diabetes complications risk profile that included clinical results from the baseline assessment and strategies to improve each measure. Results were also mailed to participants' self-identified providers. Participants were paired with PLs for one-on-one support activities outside of group sessions. During the 3-month program, PL-participant teams were expected to schedule 2 face-to-face meetings to explore motivation for making changes, identify a self-management goal, and develop an action plan. PLs were expected to make 3 follow-up telephone support calls per participant to assess participants' progress.

Ongoing Diabetes Self-Management Support (DSMS) The 12-month ongoing DSMS component (the "PLEASED intervention") was designed to provide ongoing emotional and behavioral support delivered by PLs through weekly group sessions and follow-up telephone contacts. Participants were encouraged to attend sessions as often as they needed or were able to given competing life demands. Discussion topics were guided by patients' self-management questions and concerns. While not curriculum-driven, each session addressed 5 core components:

- Reflecting on recent self-management challenges or evaluating action plans from the previous week

- Sharing feelings about these challenges and other aspects of living with diabetes

- Engaging in group-based problem-solving

- Raising questions about diabetes and its care

- Setting self-management goals and developing action plans

The PLs helped participants set goals using the 5-step behavioral goal-setting model..$^{15}$ To ensure regular contact with each participant, PLs made a telephone support call to any participant who had not attended a DSMS session in 3 consecutive weeks. The content of the telephone calls closely mirrored group-based support activities.

Participants in both study groups were encouraged to continue to receive routine care from their community-based diabetes providers. Given that participants were coming from many different health care systems, it was not feasible to assess the routine care they received using a standardized approach.

\section{Outcomes and Measures}

The primary outcome was $\mathrm{HbA}_{1 \mathrm{c}}$ level, and secondary outcomes included a lipid panel—total cholesterol, 
low-density lipoprotein cholesterol (LDL-C), and high-density lipoprotein cholesterol (HDL-C) - BP, BMI, waist circumference, and validated measures of diabetes distress and diabetes social support. Systolic and diastolic BP were taken as the average of 2 readings. All clients were weighed on a Health o meter Pro Series spring scale, and height was measured using a stadiometer. Weight and height measurements were used to calculate BMI in $\mathrm{kg} / \mathrm{m}^{2}$. Waist circumference was measured using a standard tape measure.

We measured diabetes distress with the Diabetes Distress Scale, a 17-item instrument that assesses emotional distress and functioning specific to living with diabetes, with higher scores indicating higher levels of distress. ${ }^{16}$ We assessed diabetes social support with an adapted version of the Diabetes Support Scale, a 6 -item instrument that assesses patient perceived social support as it relates to meeting emotional needs, seeking advice, and obtaining information, with higher scores indicating more support. ${ }^{17}$

\section{Statistical Analysis}

The sample size was estimated for longitudinal analysis with a linear mixed model based on a standard deviation of $1.75 \%$, or $19.1 \mathrm{mmol} / \mathrm{mol}$ and an intraclass correlation coefficient of 0.5 for repeated measures over time on the same individual. ${ }^{18}$ We calculated that with 65 subjects per group, we would have $80 \%$ power to identify a change of $0.6 \%$ in $\mathrm{HbA}_{1 \mathrm{c}}$ when comparing the 2 groups using a two-tailed $5 \%$ level of significance. To allow for $30 \%$ dropout, we aimed to recruit 93 participants per group.

In analyzing all of the outcomes, we examined whether within-group gains achieved after 3 months of the DSME program were also sustained at 15 -months following the DSMS intervention. Success of either arm in maintaining gains is indicated when $P$ values $<.05$ are observed in comparisons of baseline and follow-up values, indicating, for instance, whether improvements in $\mathrm{HbA}_{1 \mathrm{c}}$ at month 3 were sustained at months 9 and 15. We computed Spearman correlation coefficients to examine the relationship between group meeting attendance and number of support contacts on one hand and health-related improvements on the other.

All analyses were intention-to-treat. At baseline, all continuous measures except diabetes duration were compared between the PL intervention and the control condition with Student's $t$-test. Diabetes duration was analyzed with the log rank test. To check for differences between groups in categorical variables, Fisher's exact test was used if the expected count in any cell was under 5 ; Pearson's $\chi^{2}$ test was used for all other categorical variables.
All longitudinal outcomes except diabetes distress were analyzed by using a linear mixed model (LMM). Diabetes distress was analyzed using a generalized estimating equation (GEE). In the LMM, the outcome was the change in value from baseline. Both GEE and LMM allow for correlation among observations on the same person and enable participants to be included in the analysis if they had data at one or more time points. ${ }^{19-20}$ All models were adjusted for time, study group, a time-by-study-group interaction, and the baseline value. For all outcomes, the "intervention effect" was estimated by comparing the changes from baseline to follow-up in the two intervention groups.

We also conducted sensitivity analyses that included variables for diabetes, cholesterol, and BP medication intensification to ensure that intervention effects were not principally due to medications. As there was no change in the results, we report the unadjusted results.

\section{RESULTS}

\section{Participant Flow and Baseline Data}

Of the 179 individuals assessed, 27 (15\%) did not meet inclusion criteria (Figure 1). Of the 152 eligible patients, 46 (30\%) declined to participate. Among the 106 randomized patients, 78 had $\mathrm{HbA}_{1 \mathrm{c}}$ data at the 3 -month assessment; 62 at the 9 -month assessment, 64 at the 15 -month assessment (attrition rate $=40 \%$ ) (Figure 1). Loss to follow-up was not different between the 2 groups and was not associated with clinical or demographic variables. Patient characteristics are presented in Table 1. There were no significant differences in baseline characteristics between groups.

\section{Glycemic Control}

Neither the intervention nor the control group showed any change in mean $\mathrm{HbA}_{1 \mathrm{c}}$ at 3, 9, or 15 months (Table 2).

\section{Cardiovascular Risk Factors LDL Cholesterol}

The control group experienced a significant rise in mean LDL-C at 3 months $(15.5 \mathrm{mg} / \mathrm{dL}, P<.001)$ that was sustained at $9(16.5 \mathrm{mg} / \mathrm{d}, P<.001)$ and 15 months $(16.6 \mathrm{mg} / \mathrm{dL}, P=.002)$. Figure 2 presents the significant between-group differences that were seen at 3,9, and 15 months.

\section{HDL Cholesterol}

The intervention group showed significant improvements in mean HDL-C at 3 months $(5.8 \mathrm{mg} / \mathrm{dL}, P$ $<.001)$ that was sustained at 9 months $(13.6 \mathrm{mg} / \mathrm{dL}$, $P<.001)$ and 15 months $(14.1 \mathrm{mg} / \mathrm{dL}, P<.001)$. The control group did not show improvements in mean 
HDL-C at 3 months, but did at 9 months $(14.3 \mathrm{mg} / \mathrm{dL}$, $P<.001)$ and 15 months $(13.3 \mathrm{mg} / \mathrm{dL}, P<.001)$. Significant between-group differences were seen only at 3 months $(5.1 \mathrm{mg} / \mathrm{dL}, P=.005)$ in favor of the intervention group.

\section{Figure 1. CONSORT 2012 flow diagram: PLEASED Study}

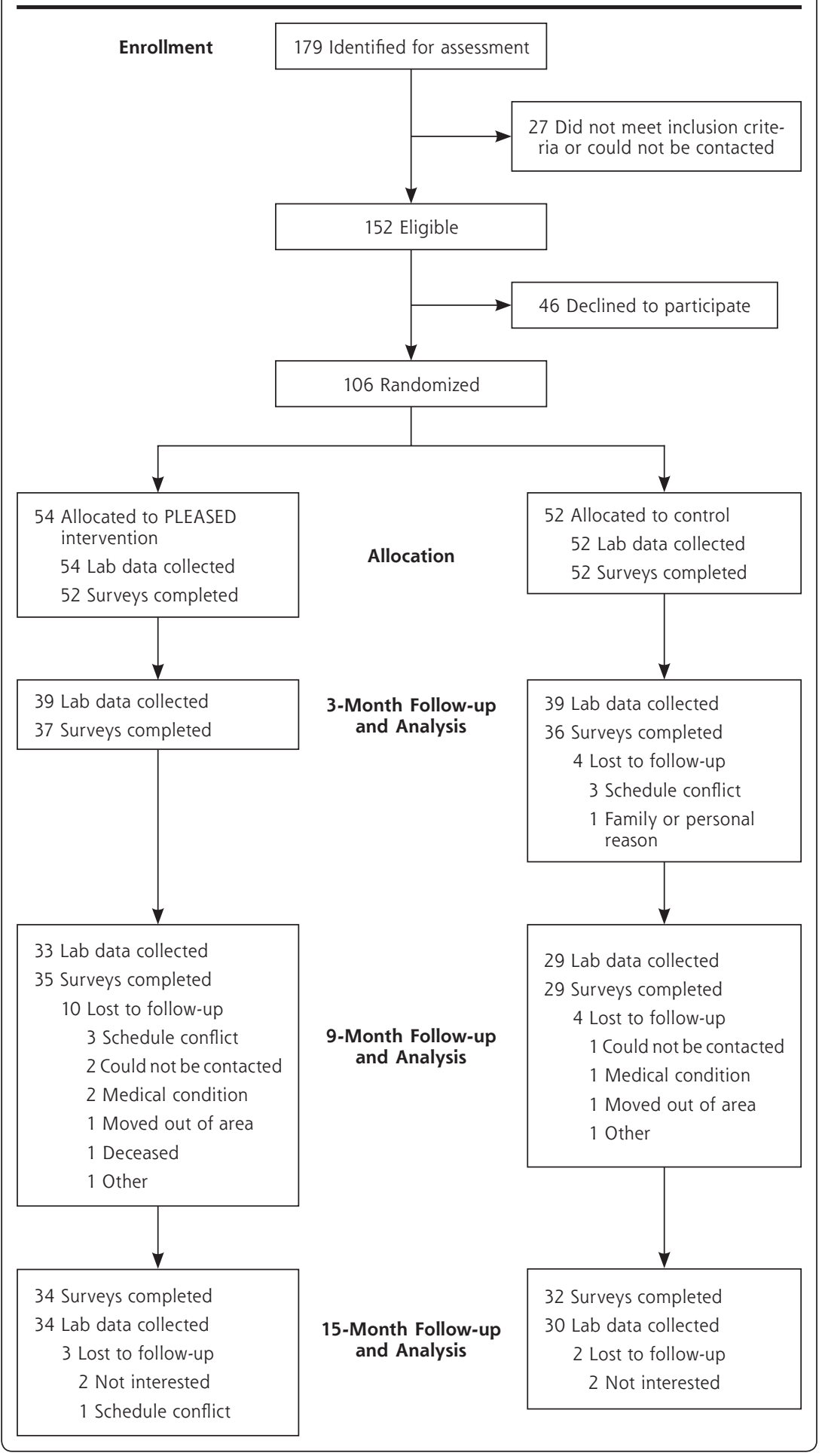

\section{Blood Pressure}

The control group experienced progressive increases in mean systolic BP over the 15 -month study period (15month increase of $7.5 \mathrm{~mm} \mathrm{Hg}$ from baseline, $P=.008$ ) leading to mean systolic BPs $10 \mathrm{~mm} \mathrm{Hg}$ higher than the PL group's mean systolic BP at 15 months $(P=.01)$. The PL group's mean diastolic BP declined at 3 months by $-4.5 \mathrm{~mm} \mathrm{Hg}(P=.007)$ from baseline, and this decline was maintained at 15 months $(-6.4$ $\mathrm{mm} \mathrm{Hg}, P=.0002)$. By 15 months, the PL group had mean diastolic BP $8.3 \mathrm{~mm} \mathrm{Hg}$ lower than that of the control group $(P=.001)$.

\section{Body Mass Index}

Mean BMI for the PL group decreased throughout the study period from baseline, sustaining a $1.0 \mathrm{~kg} / \mathrm{m}^{2}$ drop in BMI at 15 months $(P<.001)$, which was $0.8 \mathrm{~kg} / \mathrm{m}^{2}$ lower than the control group's mean at 15 months $(P=.03)$.

\section{Waist Circumference}

The PL group similarly experienced progressive reductions over the study period in waist circumference, with waist circumferences on average 1.4 inches smaller at 15 months than at baseline $(P=.003)$. However, the control group showed similar significant reductions in waist circumference at 9 and 15 months.

\section{Psychosocial Outcomes Diabetes Social Support}

From baseline to 3 months, levels of social support increased within the PL group $(0.5, P=.02)$ and were sustained at 15 months $(0.4, P=.04)$ (Table 2). While the control group did not experience changes at any point, no significant between-group differences were observed.

\section{Diabetes Distress}

Significant increases in the proportion of individuals who reported little to no diabetes dis- 
Table 1. Baseline Characteristics of Study Participants

\begin{tabular}{|c|c|c|c|c|}
\hline Characteristic & $\begin{array}{c}\text { Peer-led } \\
\text { Group } \\
(n=54)\end{array}$ & $\begin{array}{l}\text { Control } \\
\text { Group } \\
(n=52)\end{array}$ & $\begin{array}{c}\text { Total } \\
(n=106)\end{array}$ & $\begin{array}{l}\text { P Value for } \\
\text { Between- } \\
\text { Group } \\
\text { Difference }\end{array}$ \\
\hline Age, $y$, mean (SD) & $56.7(11.5)$ & $55.9(11.3)$ & $56.3(11.4)$ & $.71^{\mathrm{a}}$ \\
\hline Men, n (\%) & $17(31.5 \%)$ & $18(34.6 \%)$ & $35(33.0 \%)$ & $.73^{b}$ \\
\hline Race or ethnicity, n (\%) & & & & N/A \\
\hline African American & $54(100 \%)$ & $52(100 \%)$ & $106(100 \%)$ & \\
\hline Education, n (\%) & & & & $.33^{c}$ \\
\hline Some high school or less & $5(9.6 \%)$ & $3(5.8 \%)$ & $8(7.7 \%)$ & \\
\hline High school graduate or GED & $6(11.5 \%)$ & $7(13.5 \%)$ & $13(12.5 \%)$ & \\
\hline $\begin{array}{l}\text { Some college/technical/vocational } \\
\text { training }\end{array}$ & $25(48.1 \%)$ & $18(34.6 \%)$ & $43(41.3 \%)$ & \\
\hline College graduate or higher & $16(30.8 \%)$ & $24(46.2 \%)$ & $40(38.5 \%)$ & \\
\hline Employed, n (\%) & $20(38.5 \%)$ & $25(48.1 \%)$ & $45(43.3 \%)$ & $.32^{b}$ \\
\hline Have health insurance, n (\%) & $45(95.7 \%)$ & $49(100.0 \%)$ & $94(97.9 \%)$ & $.24^{c}$ \\
\hline Household income, n (\%) & & & & $.25^{\mathrm{b}}$ \\
\hline$<\$ 20,000$ & $9(17.3 \%)$ & $14(26.9 \%)$ & $23(22.1 \%)$ & \\
\hline$\$ 20,000-\$ 49,999$ & $21(40.4 \%)$ & $24(46.2 \%)$ & $45(43.3 \%)$ & \\
\hline$\$ 50,000$ or more & $20(38.5 \%)$ & $13(25.0 \%)$ & $33(31.7 \%)$ & \\
\hline \multicolumn{5}{|l|}{ Social support } \\
\hline Married or partnered, n (\%) & $26(50.0 \%)$ & $24(46.2 \%)$ & $50(48.1 \%)$ & $.69^{b}$ \\
\hline Diabetes social support, mean (SD) & $5.4(1.3)$ & $5.3(1.5)$ & $5.3(1.4)$ & $.73^{\mathrm{a}}$ \\
\hline Antihyperglycemic medication, n (\%) & & & & $.97^{b}$ \\
\hline No medications & $7(14.0 \%)$ & $6(12.2 \%)$ & $13(13.1 \%)$ & \\
\hline Only oral diabetes medication & $24(48.0 \%)$ & $24(49.0 \%)$ & $48(48.5 \%)$ & \\
\hline Insulin, with or without medication & $19(38.0 \%)$ & $19(38.8 \%)$ & $38(38.4 \%)$ & \\
\hline \multicolumn{5}{|l|}{ Medication adherence, n (\%) } \\
\hline $\begin{array}{l}\text { Often or very often miss insulin } \\
\text { dose/wk }\end{array}$ & $2(10.0 \%)$ & $1(4.8 \%)$ & $3(7.3 \%)$ & $.61^{c}$ \\
\hline $\begin{array}{l}\text { Often or very often miss medication } \\
\text { dose/wk }\end{array}$ & $0(0.0 \%)$ & $1(2.2 \%)$ & $1(1.3 \%)$ & $>.99^{c}$ \\
\hline \multicolumn{5}{|l|}{ General health } \\
\hline $\begin{array}{l}\text { Self-rated fair or poor general } \\
\text { health, } n(\%)\end{array}$ & $17(32.7 \%)$ & $19(36.5 \%)$ & $36(34.6 \%)$ & $.68^{b}$ \\
\hline Minimal depression, ${ }^{d} \mathrm{n}(\%)$ & $37(72.5)$ & $29(55.8)$ & $66(64.1)$ & $.08^{\mathrm{b}}$ \\
\hline Diabetes distress scale ${ }^{e}$ & & & & $.83^{b}$ \\
\hline Little or no distress & $30(57.7)$ & $33(63.5)$ & $63(60.6)$ & \\
\hline Moderate distress & $15(28.8)$ & $13(25.0)$ & $28(26.9)$ & \\
\hline High distress & $7(13.5)$ & $6(11.5)$ & $13(12.5)$ & \\
\hline \multicolumn{5}{|l|}{ Physiological measures } \\
\hline Hemoglobin $A_{1 c}, \%$, mean (SD) & $7.8(2.1)$ & $8.0(1.6)$ & $7.9(1.9)$ & $.53^{\mathrm{a}}$ \\
\hline Hemoglobin $A_{1 c}$ mmol/mol, mean (SD) & $62.0(23.0)$ & $64.0(17.5)$ & $63.0(20.8)$ & $.53^{\mathrm{a}}$ \\
\hline Total cholesterol, mg/DL, mean (SD) & $155.3(42.3)$ & $151.8(40.1)$ & $153.5(41.1)$ & $.67^{a}$ \\
\hline LDL cholesterol, mg/DL, mean (SD) & $95.3(33.8)$ & $90.1(31.8)$ & $92.7(32.8)$ & $.43^{\mathrm{a}}$ \\
\hline HDL Cholesterol, mg/DL, mean (SD) & $46.4(15.2)$ & $49.6(15.6)$ & $48.0(15.4)$ & $.28^{\mathrm{a}}$ \\
\hline Total cholesterol / HDL cholesterol & $3.5(1.0)$ & $3.2(1.0)$ & $3.4(1.0)$ & $.12^{\mathrm{a}}$ \\
\hline Systolic BP, mm Hg, mean (SD) & $137.2(19.5)$ & $137.8(18.5)$ & $137.5(18.9)$ & $.87^{\mathrm{a}}$ \\
\hline Diastolic BP, mm Hg, mean (SD) & $87.5(13.2)$ & $86.4(13.4)$ & $87.0(13.2)$ & $.69^{a}$ \\
\hline Body mass index, $\mathrm{kg} / \mathrm{m}^{2}$, mean (SD) & $36.2(7.4)$ & $35.4(8.5)$ & $35.8(7.9)$ & $.59^{a}$ \\
\hline Waist circumference, inches, mean (SD) & $44.9(6.3)$ & $45.3(6.6)$ & $45.1(6.4)$ & $.73^{\mathrm{a}}$ \\
\hline Diabetes duration, years, mean (SD) & $8.0(6.9)$ & $10.5(9.7)$ & $9.2(8.5)$ & $.14^{f}$ \\
\hline \multicolumn{5}{|c|}{$\begin{array}{l}\text { a } t \text {-Test. } \\
\text { b Pearson } \chi^{2} \text { test. } \\
\text { ' Fisher's exact test. } \\
\text { d Minimal depression indicated by PHQ (Primary Care Evaluation of Mental Disorders) } \geq 3 \text {. } \\
\text { e Diabetes Distress Scale (DDS); DDS <2: little or no distress; } 2 \leq \text { DDS <3: moderate distress; DDS } \geq 3 \text { : high distress. } \\
{ }^{f} \text { Log-Rank test. }\end{array}$} \\
\hline
\end{tabular}

tress occurred at 3 months in each group (PL 58.1\% at baseline vs $74.4 \%$ at 3 months, $P=.01$; Control $63.5 \%$ vs $90.2 \%, P=.01$ ). While the PL group did not sustain the reduced diabetes distress levels after 3 months, the control group maintained $83.7 \%$ $(P=.03)$ at 15 months.

\section{Relationship Between Participation Level and Health-Related Improvements}

No associations were observed between session attendance or number of contacts outside of sessions and clinical improvements. However, support group attendance and number of contacts were correlated with the maintenance of lower diabetes distress at 15 -months $(\mathrm{r}=-0.448, P=.009$ and $r=-0.373, P=.03$, respectively). After adjusting for attendance and support contacts, results remained the same for all primary and secondary outcomes.

\section{DISCUSSION}

Although the PLEASED intervention had little impact on glycemic control, this peer support model did have a positive or stabilizing effect on several important cardiovascular disease (CVD) risk factors. Specifically, improvements in HDL-C, diastolic $\mathrm{BP}$, and waist circumference achieved at 3 months were sustained at 15 months. Furthermore, while the control group experienced an initial and sustained increase in 
Table 2. Changes in Clinical and Psychological Outcomes Over 15-Month Follow-Up

\begin{tabular}{|c|c|c|c|c|c|}
\hline Outcome & Group & Baseline & 3 Months-Baseline & 9 Months-Baseline & 15 Months-Baseline \\
\hline \multirow[t]{3}{*}{$\mathrm{HbA}_{\mathrm{lc}} \%$, mean $(\mathrm{Cl})$} & Intervention & $\begin{array}{c}7.8 \\
(7.2-8.4)\end{array}$ & $\begin{array}{c}-0.1 \\
(-0.4 \text { to } 0.3) P=.72\end{array}$ & $\begin{array}{c}0.0 \\
(-0.4 \text { to } 0.4) P>.99\end{array}$ & $\begin{array}{c}0.5 \\
(-0.1 \text { to } 1.1) P=.10\end{array}$ \\
\hline & Control & $\begin{array}{c}8.0 \\
(7.6-8.5)\end{array}$ & $\begin{array}{c}-0.3 \\
(-0.7 \text { to } 0.0) P=.08\end{array}$ & $\begin{array}{c}0.1 \\
(-0.3 \text { to } 0.5) P=.61\end{array}$ & $\begin{array}{c}0.1 \\
(-0.5 \text { to } 0.8) P=.71\end{array}$ \\
\hline & $\begin{array}{c}\text { Control } \\
\text { vs Intervention }\end{array}$ & $\begin{array}{c}-0.2 \\
(-1.0 \text { to } 0.5) \\
P=.53\end{array}$ & $\begin{array}{c}0.3 \\
(-0.2 \text { to } 0.8) \\
P=.30\end{array}$ & $\begin{array}{c}-0.1 \\
(-0.7 \text { to } 0.5) \\
P=.71\end{array}$ & $\begin{array}{c}0.4 \\
(-0.5 \text { to } 1.3) \\
P=.39\end{array}$ \\
\hline \multirow[t]{3}{*}{$\begin{array}{l}\mathrm{HbA}_{1 c}, \mathrm{mmol} / \mathrm{mol} \\
\text { mean, (Cl) }\end{array}$} & Intervention & $\begin{array}{c}62.0 \\
(55.0-68.0)\end{array}$ & $\begin{array}{c}-1.1 \\
(-4.4 \text { to } 3.3) P=.72\end{array}$ & $\begin{array}{c}0.0 \\
(-4.4 \text { to } 4.4) P>.99\end{array}$ & $\begin{array}{c}5.5 \\
(-1.1 \text { to } 12.0) P=.10\end{array}$ \\
\hline & Control & $\begin{array}{c}64.0 \\
(60.0-69.0)\end{array}$ & $\begin{array}{c}-3.3 \\
(-7.7 \text { to } 0.0) P=.07\end{array}$ & $\begin{array}{c}1.1 \\
(-3.3 \text { to } 5.5) P=.61\end{array}$ & $\begin{array}{c}1.1 \\
(-5.5 \text { to } 8.7) P=.71\end{array}$ \\
\hline & $\begin{array}{l}\text { Control } \\
\text { vs Intervention }\end{array}$ & $\begin{array}{c}-2.2 \\
(-10.9 \text { to } 5.5) \\
P=.53\end{array}$ & $\begin{array}{c}3.3 \\
(-2.2 \text { to } 8.7) \\
P=.30\end{array}$ & $\begin{array}{c}-1.1 \\
(-7.7 \text { to } 5.5) \\
P=.71\end{array}$ & $\begin{array}{c}4.4 \\
(-5.5 \text { to } 14.2) \\
P=.39\end{array}$ \\
\hline \multirow[t]{3}{*}{$\begin{array}{l}\text { LDL cholesterol, } \\
\text { mg/dL, mean (Cl) }\end{array}$} & Intervention & $\begin{array}{c}95.3 \\
(85.8-104.7)\end{array}$ & $\begin{array}{c}3.0 \\
(-3.8 \text { to } 9.9) P=.39\end{array}$ & $\begin{array}{c}4.6 \\
(-2.6 \text { to } 11.8) P=.22\end{array}$ & $\begin{array}{c}1.6 \\
(-7.7 \text { to } 10.9) P=.74\end{array}$ \\
\hline & Control & $\begin{array}{c}90.1 \\
(81.3-99.0)\end{array}$ & $\begin{array}{c}15.5 \\
(8.6-22.3) P<.001\end{array}$ & $\begin{array}{c}16.5 \\
(8.9-24.1) P<.001\end{array}$ & $\begin{array}{c}16.6 \\
(6.8-26.3) P=.002\end{array}$ \\
\hline & $\begin{array}{l}\text { Control } \\
\text { vs Intervention }\end{array}$ & $\begin{array}{c}5.1 \\
(-7.6 \text { to } 17.9) \\
P=.43\end{array}$ & $\begin{array}{c}-12.5 \\
(-22.1 \text { to }-2.8) P=.01\end{array}$ & $\begin{array}{c}-11.9 \\
(-22.4 \text { to }-1.5) P=.03\end{array}$ & $\begin{array}{c}-15.0 \\
(-28.5 \text { to }-1.5) P=.03\end{array}$ \\
\hline \multirow[t]{3}{*}{$\begin{array}{l}\text { HDL cholesterol, } \\
\text { mg/dL, mean }(\mathrm{Cl})\end{array}$} & Intervention & $\begin{array}{c}46.3 \\
(42.1-50.6)\end{array}$ & $\begin{array}{c}5.8 \\
(3.4-8.3) P<.001\end{array}$ & $\begin{array}{c}13.6 \\
(10.3-16.9) P<.001\end{array}$ & $\begin{array}{c}14.1 \\
(9.9-18.2) P<.001\end{array}$ \\
\hline & Control & $\begin{array}{c}49.6 \\
(45.3-54.0)\end{array}$ & $\begin{array}{c}0.8 \\
(-1.7 \text { to } 3.2) P=.54\end{array}$ & $\begin{array}{c}14.3 \\
(10.8-17.8) P<.001\end{array}$ & $\begin{array}{c}13.3 \\
(8.9-17.7) P<.001\end{array}$ \\
\hline & $\begin{array}{l}\text { Control } \\
\text { vs Intervention }\end{array}$ & $\begin{array}{c}-3.3 \\
(-9.3 \text { to } 2.7) \\
P=.28\end{array}$ & $\begin{array}{c}5.1 \\
(1.7-8.5) \\
P=.005\end{array}$ & $\begin{array}{c}-0.7 \\
(-5.5 \text { to } 4.1) \\
P=.78\end{array}$ & $\begin{array}{l}0.8 \\
(-5.3 \text { to } 6.8) \\
P=.81\end{array}$ \\
\hline \multirow{3}{*}{$\begin{array}{l}\text { Systolic blood pres- } \\
\text { sure, } \mathrm{mm} \mathrm{Hg} \text {, } \\
\text { mean (Cl) }\end{array}$} & Intervention & $\begin{array}{c}137.2 \\
(131.8-142.5)\end{array}$ & $\begin{array}{c}-0.7 \\
(-5.6 \text { to } 4.2) P=.78\end{array}$ & $\begin{array}{c}-0.6 \\
(-6.0 \text { to } 4.8) P=.83\end{array}$ & $\begin{array}{c}-2.5 \\
(-7.7 \text { to } 2.7) P=.35\end{array}$ \\
\hline & Control & $\begin{array}{c}137.8 \\
(132.6-142.9)\end{array}$ & $\begin{array}{c}3.6 \\
(-1.3 \text { to } 8.6) P=.15\end{array}$ & $\begin{array}{c}4.3 \\
(-1.2 \text { to } 9.9) P=.13\end{array}$ & $\begin{array}{c}7.5 \\
(2.0-13.0) P=.008\end{array}$ \\
\hline & $\begin{array}{l}\text { Control } \\
\text { vs Intervention }\end{array}$ & $\begin{array}{c}-0.6 \\
(-7.9 \text { to } 6.7) \\
P=.87\end{array}$ & $\begin{array}{c}-4.3 \\
(-11.3 \text { to } 2.6) \\
P=.23\end{array}$ & $\begin{array}{c}-4.9 \\
(-12.7 \text { to } 2.8) \\
P=.22\end{array}$ & $\begin{array}{c}-10.0 \\
(-17.6 \text { to }-2.4) \\
P=.01\end{array}$ \\
\hline \multirow{3}{*}{$\begin{array}{l}\text { Diastolic blood } \\
\text { pressure, mm Hg, } \\
\text { mean (Cl) }\end{array}$} & Intervention & $\begin{array}{c}87.4 \\
(83.8-91.1)\end{array}$ & $\begin{array}{c}-4.5 \\
(-7.7 \text { to }-1.3) P=.007\end{array}$ & $\begin{array}{c}-3.3 \\
(-6.7 \text { to } 0.1) P=.06\end{array}$ & $\begin{array}{c}-6.4 \\
(-9.8 \text { to }-3.1) P<.001\end{array}$ \\
\hline & Control & $\begin{array}{c}86.4 \\
(82.7-90.2)\end{array}$ & $\begin{array}{c}1.9 \\
(-1.3 \text { to } 5.1) P=.26\end{array}$ & $\begin{array}{c}1.6 \\
(-2.0 \text { to } 5.1) P=.39\end{array}$ & $\begin{array}{c}1.9 \\
(-1.7 \text { to } 5.4) P=.31\end{array}$ \\
\hline & $\begin{array}{l}\text { Control } \\
\text { vs Intervention }\end{array}$ & $\begin{array}{c}1.0 \\
(-4.1 \text { to } 6.1) \\
P=.69\end{array}$ & $\begin{array}{c}-6.4 \\
(-10.9 \text { to }-1.8) \\
P=.007\end{array}$ & $\begin{array}{c}-4.8 \\
(-9.8 \text { to } 0.1) \\
P=.06\end{array}$ & $\begin{array}{c}-8.3 \\
(-13.2 \text { to }-3.4) \\
P=.001\end{array}$ \\
\hline \multirow[t]{3}{*}{$\begin{array}{l}\mathrm{BMI}, \mathrm{kg} / \mathrm{m}^{2} \\
\text { mean }(\mathrm{Cl})\end{array}$} & Intervention & $\begin{array}{c}36.2 \\
(34.2-38.3)\end{array}$ & $\begin{array}{l}\quad-0.4 \\
(-0.9 \text { to } 0.1) P P=.12\end{array}$ & $\begin{array}{c}-0.7 \\
(-1.2 \text { to }-0.1) P=.01\end{array}$ & $\begin{array}{c}-1.0 \\
(-1.5 \text { to }-0.5) P<.001\end{array}$ \\
\hline & Control & $\begin{array}{c}35.4 \\
(33.1-37.8)\end{array}$ & $\begin{array}{c}-0.2 \\
(-0.7 \text { to } 0.3) P=.42\end{array}$ & $\begin{array}{c}-0.3 \\
(-0.8 \text { to } 0.3) P=.35\end{array}$ & $\begin{array}{c}-0.2 \\
(-0.7 \text { to } 0.3) P=.49\end{array}$ \\
\hline & $\begin{array}{l}\text { Control } \\
\text { vs Intervention }\end{array}$ & $\begin{array}{c}0.8 \\
(-2.2 \text { to } 3.9) \\
P=.59\end{array}$ & $\begin{array}{c}-0.2 \\
(-0.9 \text { to } 0.5) \\
P=.60\end{array}$ & $\begin{array}{c}-0.4 \\
(-1.2 \text { to } 0.3) \\
P=.29\end{array}$ & $\begin{array}{c}-0.8 \\
(-1.6 \text { to }-0.1) \\
P=.03\end{array}$ \\
\hline \multirow[t]{3}{*}{$\begin{array}{l}\text { Waist circumference, } \\
\text { inches, mean (Cl) }\end{array}$} & Intervention & $\begin{array}{c}44.8 \\
(43.1-46.6)\end{array}$ & $\begin{array}{c}-1.0 \\
(-1.8 \text { to }-0.2) P=.02\end{array}$ & $\begin{array}{c}-1.3 \\
(-2.2 \text { to }-0.5) P=.003\end{array}$ & $\begin{array}{c}-1.4 \\
(-2.2 \text { to }-0.5) P=.003\end{array}$ \\
\hline & Control & $\begin{array}{c}45.3 \\
(43.4-47.1)\end{array}$ & $\begin{array}{c}-0.7 \\
(-1.5 \text { to } 0.1) P=.10\end{array}$ & $\begin{array}{c}-1.4 \\
(-2.3 \text { to }-0.5) P=.003\end{array}$ & $\begin{array}{c}-1.3 \\
(-2.2 \text { to }-0.4) P=.004\end{array}$ \\
\hline & $\begin{array}{l}\text { Control } \\
\text { vs Intervention }\end{array}$ & $\begin{array}{c}-0.4 \\
(-2.9 \text { to } 2.1) \\
P=.73\end{array}$ & $\begin{array}{c}-0.3 \\
(-1.5 \text { to } 0.9) \\
P=.61\end{array}$ & $\begin{array}{c}0.0 \\
(-1.2 \text { to } 1.3) \\
P=.95\end{array}$ & $\begin{array}{c}0.0 \\
(-1.3 \text { to } 1.2) \\
P=.98\end{array}$ \\
\hline
\end{tabular}

LDL-C and systolic blood pressure, these clinical endpoints remained constant throughout the intervention for the PL group, which left the PL group with sig- nificantly better levels of these measures at 15 months than the control group.

A striking finding of our study is the lack of any 
Table 2. Changes in Clinical and Psychological Outcomes Over 15-Month Follow-Up, continued

\begin{tabular}{|c|c|c|c|c|c|}
\hline Outcome & Group & Baseline & 3 Months-Baseline & 9 Months-Baseline & 15 Months-Baseline \\
\hline \multirow[t]{3}{*}{$\begin{array}{l}\text { Diabetes support } \\
\text { scale, mean (CI) }\end{array}$} & Intervention & $\begin{array}{c}5.4 \\
(5.0-5.7)\end{array}$ & $\begin{array}{c}0.5 \\
(0.1-0.9) P=.02\end{array}$ & $\begin{array}{c}0.2 \\
(-0.2 \text { to } 0.6) P=.33\end{array}$ & $\begin{array}{c}0.4 \\
(0.0-0.9) P=.04\end{array}$ \\
\hline & Control & $\begin{array}{c}5.3 \\
(4.8-5.7)\end{array}$ & $\begin{array}{c}0.4 \\
(0.0-0.8) P=.07\end{array}$ & $\begin{array}{c}0.4 \\
(0.0-0.8) P=.08\end{array}$ & $\begin{array}{c}0.2 \\
(-0.2 \text { to } 0.6) P=.31\end{array}$ \\
\hline & $\begin{array}{l}\text { Control } \\
\text { vs Intervention }\end{array}$ & $\begin{array}{c}0.1 \\
(-0.5 \text { to } 0.7) \\
P=.73\end{array}$ & $\begin{array}{c}0.1 \\
(-0.5 \text { to } 0.7) \\
P=.75\end{array}$ & $\begin{array}{c}-0.2 \\
(-0.8 \text { to } 0.4) \\
P=.53\end{array}$ & $\begin{array}{c}0.2 \\
(-0.4 \text { to } 0.8) \\
P=.48\end{array}$ \\
\hline \multirow[t]{3}{*}{$\begin{array}{l}\text { Little to no diabetes } \\
\text { distress, a \% (CI) }\end{array}$} & Intervention & $\begin{array}{c}58.1 \\
(45-71)\end{array}$ & $\begin{array}{c}74.4 \\
(59-85) P=.01\end{array}$ & $\begin{array}{c}69.5 \\
(55-81) P=.08\end{array}$ & $\begin{array}{c}64.0 \\
(50-76) P=.30\end{array}$ \\
\hline & Control & $\begin{array}{c}63.5 \\
(50-75)\end{array}$ & $\begin{array}{c}90.2 \\
(74-97) P=.01\end{array}$ & $\begin{array}{c}85.0 \\
(68-94) P=.04\end{array}$ & $\begin{array}{c}83.7 \\
(67-93) P=.03\end{array}$ \\
\hline & $\begin{array}{l}\text { Control } \\
\text { vs Intervention }\end{array}$ & $\begin{array}{c}-5.3 \\
(-24 \text { to } 13) \\
P=.58\end{array}$ & $\begin{array}{c}-10.5 \\
(-32 \text { to } 11) \\
P=.19\end{array}$ & $\begin{array}{c}-10.1 \\
(-33 \text { to } 12) \\
P=.29\end{array}$ & $\begin{array}{c}-14.4 \\
(-37 \text { to } 9) \\
P=.13\end{array}$ \\
\hline \multicolumn{6}{|c|}{$\mathrm{BMI}=$ body mass index; $\mathrm{Cl}=95 \%$ confidence interval. } \\
\hline \multicolumn{6}{|c|}{$\begin{array}{l}\text { Note: } N=106 \text { : Intervention } n=54 \text {, Control } n=52 \text {. All longitudinal outcomes except diabetes distress were analyzed using a linear mixed model (LMM). Diabetes dis- } \\
\text { tress was analyzed using a generalized estimating equation (GEE). Control-vs-Intervention } P \text { values are between-group values; other } P \text { values given are for within-group } \\
\text { difference from baseline to the specified time. }\end{array}$} \\
\hline \multicolumn{6}{|c|}{$\begin{array}{l}\text { a Diabetes Distress Scale (DDS). DDS }<2 \text { : little or no distress; } 2 \leq \text { DDS }<3 \text { : moderate distress; DDS } \geq 3 \text { : high distress. P values for DDS are for change relative to baseline } \\
\text { Due to sparse cell counts in the "High Diabetes Distress" category at follow-up times, the logistic regression categories were "Little to no Diabetes Distress" vs "Moder- } \\
\text { ate to High Diabetes Distress." For the method used to determine confidence intervals for diabetes distress scores, see Supplemental Appendix at http://www.annfa- } \\
\text { mmed.org/content/13/Suppl_1/S27/suppl/DC1. }\end{array}$} \\
\hline \multicolumn{6}{|c|}{ b Minimal depression indicated by PHQ (Primary Care Evaluation of Mental Disorders) $\geq 3$. } \\
\hline
\end{tabular}

change in $\mathrm{HbA}_{1 \mathrm{c}}$ either through the 3-month DSME program or over the following 12 -month period in either study group. These findings differ from those of a previous study testing the same peer support model among Latino adults recruited from a clinic. ${ }^{21}$ In that study, $\mathrm{HbA}_{1 \mathrm{c}}$ levels in the peer support group dropped significantly by the end of 6 months of communityhealth-worker-led DSME, and the improvement was sustained at 18 months. These studies did differ in key respects. In the previous investigation, the DSME component was more intensive and occurred over a longer period ${ }_{i}$ also, baseline $\mathrm{HbA}_{1 \mathrm{c}}$ levels among participants were higher. Furthermore, the DSME delivery style for the Latino community was more structured and topic-led than the more fluid, patientdirected delivery style used in this study for the African American community. Although this problembased method was successfully used in a previous study among urban African Americans, ${ }^{22}$ the goodness of fit between teaching approaches and learning styles could play a significant role in intervention success, and the optimal fit likely differs across sociocultural groups in different settings. ${ }^{23}$

The few peer support studies that have examined long-term maintenance of short-term gains have reported positive results, but largely for psychosocial outcomes. ${ }^{24-25}$ Adding to this body of literature, our study provides evidence for the long-term maintenance of CVD improvements achieved through a short-term DSME program. In the absence of any intervention,
CVD risk factors such as LDL-C and systolic blood pressure tend to worsen over time. ${ }^{26}$ On average, this peer support model helped prevent this progressive deterioration from occurring. In the UKPDS study, achieving BP levels lower than 140/90 mm Hg led to an absolute risk reduction of $11 \%$ in diabetes complications over 10 years, an effect 3.5 times greater than that of intensive blood glucose control. ${ }^{27}$ While intensive glycemic control has an incremental cost-effectiveness of $\$ 40,000$ to $\$ 50,000$ per quality-adjusted life-year (QALY), intensive BP control actually saves almost $\$ 2,000$ per QALY. ${ }^{28-29}$ To our knowledge, this is the first study to demonstrate the stabilizing effect of peer support on these critically important outcomes.

That social support ratings increased early on and were sustained for the PL group is not surprising. Relatively unexpected, however, was the sustained improvements in diabetes distress for the control group, but not the PL group. Considering that our measure of diabetes distress encompassed multiple dimensions, there may have been aspects of distress (eg, physician-related distress) on which our peer support model exerted little to no influence. These counterintuitive findings require further investigation.

This study has several limitations. First, because the intervention was a community-based program that recruited self-selected participants, our sample was likely more motivated than one recruited from a clinic database. Second, given that participants received care from providers affiliated with many different health 


\section{Figure 2. Trajectory of unadjusted mean LDL cholesterol levels over time}

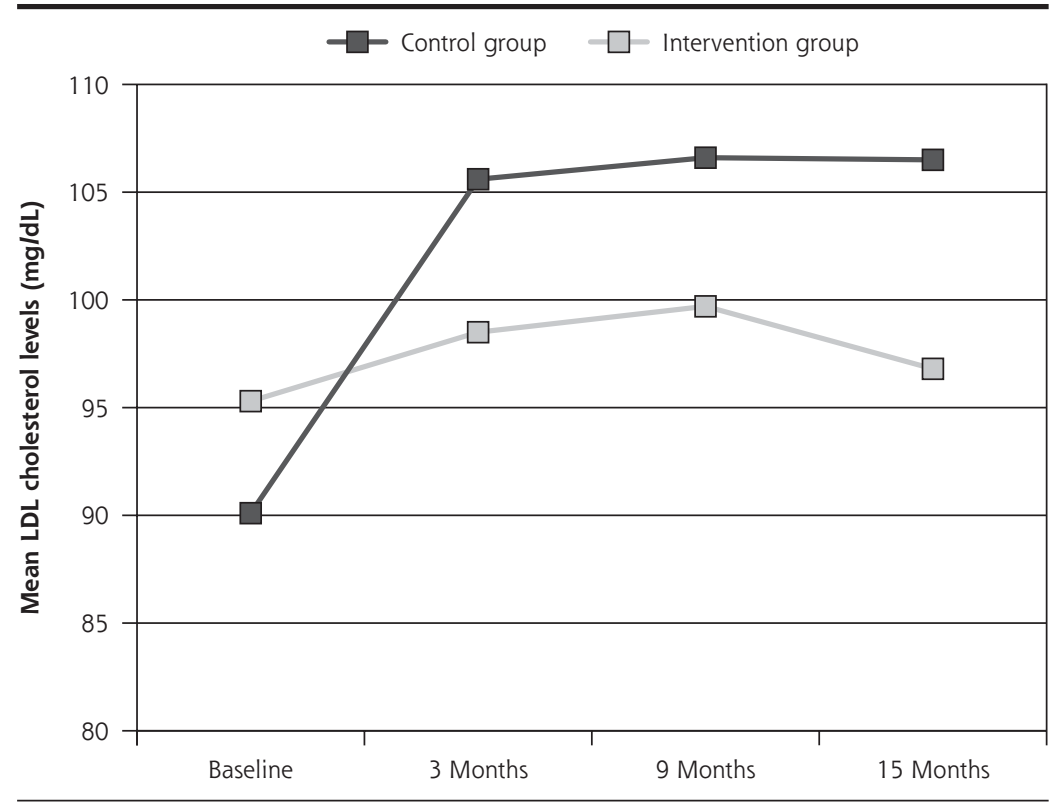

Note: At baseline, LDL cholesterol (LDL-C) levels did not differ significantly between groups. All increases within the Control group were significant $(P<.05)$ relative to the Intervention group. Within-group changes from baseline to 3,9 , and 15 months were all significant for the Control group $(P=.01)$. management support. It is important, however, to acknowledge the wide variation across peer support models as well as the sociocultural and economic diversity of the communities where these models are applied. Additional studies are clearly needed to determine the most efficacious and appealing models of peer support in order to improve outcomes among underserved people with diabetes and their family members.

\section{To read or post commentaries in response to this article, see it online at http://www.annfammed.org/content/13/ Suppl_1/S27.}

Submitted March 3, 2015; submitted, revised May 4, 2015; accepted June 4, 1015.

Key words: diabetes; peer support; health care disparities; randomized controlled trial; blood glucose control; self-management

Trials registration: ClinicalTrials.gov Protocol Record DRDA 90-0893 care systems and clinics, it was not feasible to assess or standardize follow-up care. However, randomization rendered the variation in "routine care" the same for both control and intervention groups. Third, our follow-up rate of $60 \%$ was lower than the $67 \%$ to $85 \%$ range reported in other studies targeting the African American community. ${ }^{10-13}$ While this 15 -month intervention was lengthier ${ }^{11-13}$ than some other studies, our low retention rate still raises questions concerning acceptability and sustainability. Towards the end of the intervention, some participants appeared to experience intervention "burnout." Perhaps these individuals derived the most benefit from active face-to-face and telephone support in the short-term but did not require this level of intensity over the long-term. Future peer support interventions should consider offering a range of communication modalities (eg, email and text messaging in addition to face-to-face and telephone encounters) to better accommodate variations in the amount and intensity of support needs. Although peer leader burnout was not related to participant dropout, we should minimize it by increasing our peer leader network and defraying the time investment across a larger number of volunteers.

As the number of people with diabetes grows exponentially, particularly in low-resource communities, the evidence continues to grow for peer support as a viable and compelling approach to lifelong diabetes self-
Acknowledgments: The Principal Investigators, Tricia Tang and Michele Heisler, had full access to all of the data in the study and take responsibility for the integrity of the data and accuracy of the data analysis. We thank the very dedicated peer leaders who participated in this intervention, the Ann Arbor Community Center in Ann Arbor and the Parkside Community Center in Ypsilanti, Michigan.

Funding support: Funding for this research was provided by the American Academy of Family Physicians Foundation through the Peers for Progress program with support from the Eli Lilly and Company Foundation and by the National Institute of Diabetes and Digestive and Kidney Diseases (Grant Number R18DK0785501A1). The funding sources had no role in the study design; data collection; administration of the interventions; analysis, interpretation, or reporting of data; or decision to submit the findings for publication.

Supplementary materials: Available at http://www.AnnFamMed. org/content/13/Suppl_1/S27/suppl/DC1/

\section{References}

1. Center for Disease Control and Prevention. National Diabetes Statistics Report: Estimates of Diabetes and its Burden in the United States, 2014. Atlanta, GA: US Department of Health and Human Services; 2014.

2. Heisler M, Faul JD, Hayward RA, Langa KM, Blaum C, Weir D. Mechanisms for racial and ethnic disparities in glycemic control in middle-aged and older Americans in the health and retirement study. Arch Intern Med. 2007;167(17):1853-1860.

3. Kirk JK, D’Agostino RB Jr, Bell RA, et al. Disparities in HbA1c levels between African-American and non-Hispanic white adults with diabetes: a meta-analysis. Diabetes Care. 2006;29(9):2130-2136.

4. Ford ES. Trends in the control of risk factors for cardiovascular disease among adults with diagnosed diabetes: findings from the National Health and Nutrition Examination Survey 1999-2008. J Diabetes. 2011;3(4):337-347. 
5. Egede LE, Gebregziabher M, Lynch CP, Gilbert GE, Echols C. Longitudinal ethnic differences in multiple cardiovascular risk factor control in a cohort of US adults with diabetes. Diabetes Res Clin Pract. 2011;94(3):385-394.

6. Oster NV, Welch V, Schild L, Gasmararian J, Rask K, Spettell C. Differences in self-management and use of preventive services behaviors among diabetes management enrollees by race and ethnicity. Dis Manag. 2006;9(3):167-175.

7. Dale JR, Williams SM, Bowyer V. What is the effect of peer support on diabetes outcomes in adults? A systematic review. Diabet Med. 2012;29(11):1361-1377.

8. Pfeiffer PN, Heisler M, Piette JD, Rogers MAM, Valenstein M. Efficacy of peer support interventions for depression: a meta-analysis. Gen Hosp Psychiatry. 2011;33(1):29-36.

9. Keyserling TC, Samuel-Hodge CD, Ammerman AS, et al. A randomized trial of an intervention to improve self-care behaviors of African-American women with type 2 diabetes: impact on physical activity. Diabetes Care. 2002;25(9):1576-1583.

10. Murrock CJ, Higgins PA, Killion C. Dance and peer support to improve diabetes outcomes in African American women. Diabetes Educ. 2009;35(6):995-1003.

11. Anderson-Loftin W, Barnett S, Bunn P, Sullivan P, Hussey J, Tavakoli A. Soul food light: culturally competent diabetes education. Diabetes Educ. 2005;31(4):555-563.

12. Shaya FT, Chirikov VV, Howard D, et al. Effect of social networks intervention in type 2 diabetes: a partial randomized study. J Epidemiol Commun. H 2014; 68(4):326-332.

13. Long JA, Jahnle EC, Richardson DM, Loewenstein G, Volpp KG. Peer mentoring and financial incentives to improve glucose control in African American veterans: a randomized trial. Ann Intern Med. 2012;156(6):416-424.

14. Tang TS, Funnell MM, Gillard M, Nwankwo R, Heisler M. Training peers to provide ongoing diabetes self-management support (DSMS): results from a pilot study. Patient Educ Couns. 2011;85(2):160-168.

15. Anderson RM, Funnell MM. Patient empowerment: myths and misconceptions. Patient Educ Couns. 2010;79(3):277-282.

16. Polonsky WH, Fisher L, Earles J, et al. Assessing psychosocial distress in diabetes: development of the diabetes distress scale. Diabetes Care. 2005;28(3):626-631.

17. Barrera M Jr, Glasgow RE, McKay HG, Boles SM, Feil EG. Do Internet-based support interventions change perceptions of social support?: An experimental trial of approaches for supporting diabetes self-management. Am J Community Psychol. 2002;30(5):637-654.
18. Diggle PJ, Heagerty P, Liang K, Zeger SL. Analysis of Longitudinal Data. New York, NY: Oxford University Press; 2002.

19. Little RJA, Rubin DB. Statistical Analysis With Missing Data. 2nd edition, New York, NY: John Wiley \& Sons; 2002.

20. Rubin DB. Multiple Imputation for Nonresponse in Surveys. New York, NY: John Wiley \& Sons; 1987.

21. Tang TS, Funnell $M$, Sinco $B$, et al. Comparative effectiveness of peer leaders and community health workers in diabetes selfmanagement support: results of a randomized controlled trial. Diabetes Care. 2014;37(6):1525-1534.

22. Anderson RM, Funnell MM, Nwankwo R, Gillard ML, Oh M, Fitzgerald JT. Evaluating a problem-based empowerment program for African Americans with diabetes: results of a randomized controlled trial. Ethn Dis. 2005;15(4):671-678.

23. Tang TS, Sohal PS, Garg AK. Rethinking peer support for diabetes in Vancouver's South-Asian community: a feasibility study. Diabet Med. In press 10.1111/dme.12655.

24. Lorig K, Ritter PL, Villa F, Piette JD. Spanish diabetes selfmanagement with and without automated telephone reinforcement: two randomized trials. Diabetes Care. 2008;31(3):408-414.

25. Lorig K, Ritter PL, Villa FJ, Armas J. Community-based peer-led diabetes self-management: a randomized trial. Diabetes Educ. 2009; 35(4):641-651.

26. Heisler M, Vijan S, Makki F, Piette JD. Diabetes control with reciprocal peer support versus nurse care management: a randomized trial. Ann Intern Med. 2010;153(8):507-515.

27. UK Prospective Diabetes Study (UKPDS) Group. Intensive bloodglucose control with sulphonylureas or insulin compared with conventional treatment and risk of complications in patients with type 2 diabetes (UKPDS 33). Lancet. 1998;352(9131):837-853.

28. CDC Diabetes Cost-effectiveness Group. Cost-effectiveness of intensive glycemic control, intensified hypertension control, and serum cholesterol level reduction for type 2 diabetes. JAMA. 2002;287(19): 2542-2551.

29. Zhang P, Engelgau MM, Norris SL, Gregg EW, Narayan KM. Application of economic analysis to diabetes and diabetes care. Ann Intern Med. 2004;140(11):972-977. 